\title{
ESTIMATION OF CAST IRON SUBSTITUTE THERMAL CAPACITY USING THE EXPERIMENTAL DATA
}

\begin{abstract}
In the paper the problem of the cast iron substitute thermal capacity estimation is discussed. This parameter appears when the macroscopic mathematical model of alloys solidification bases on the one domain method (fixed domain approach). In the case of cast iron the form of function describing the course of temperature-dependent thermal capacity is quite complex. Using the experimental data, in particular the measured cooling/heating curves at the set of points selected in the casting - mould domain the identification problem has been solved using the gradient methods. The results presented concern the gray iron $3.21 \% \mathrm{C}$ and $1.9 \% \mathrm{Si}$.

Keywords: modeling of alloys solidification, numerical methods, sensitivity analysis, inverse problems.
\end{abstract}

\section{Introduction}

Most practical problems associated with the heat conduction modeling are currently solved using the numerical methods, but the reliability of the results depends on the proper adoption of the input data, including the parameters appearing in the mathematical description of the process considered. In the case of the models basing on the Fourier-type equation should be mentioned here the volumetric specific heat and thermal conductivity of material, the capacity of internal heat sources and the parameters determining the form of boundaryinitial conditions. They can be treated as the constant values or, usually, as the temperature - dependent functions.

The very popular approach to the description of alloys solidification in the case of macroscale modeling is the application of the energy equation known in literature as the one domain method [1-5]. In this equation the parameter called 'a substitute thermal capacity' (STC) appears. For the molten metal and solidified part of the casting the substitute thermal capacity corresponds directly to the volumetric specific heats of subdomains considered, while in the mushy zone region the evolution of latent heat determined the final form of the parameter discussed. Taking into account the complex mechanism of cast iron solidification the substitute thermal capacity must be defined in an unusual way. The preliminary studies using the methods of thermal and differential analysis (TDA) [6] shown that the good approximation of STC can be obtained by the composition of two bell-type functions and the constant value between them. The detailed form of the parameter discussed has been found using the methods of inverse problems solution [7-13], in particular, the gradient method has been applied $[10,11]$. At the stage of numerical computations the explicit scheme of finite difference method [14-17] for non-linear heat conduction problems has been used.
In the paper the mathematical description of the solidification and cooling processes proceeding in the casting domain are discussed, next the considerations concerning the STC construction are presented. The most essential information about the gradient method and the results of testing computations are collected in the chapter 4. Chapter 5 is devoted to the application of real measurements for STC parameters estimation and finally the conclusions are formulated.

\section{One domain (fixed domain) method}

The name 'one domain method' concerns the mathematical model of alloys solidification in which the evolution of latent heat is taken into account by introducing into the energy equation the parameter called 'a substitute thermal capacity'. So, we consider the following Fourier type equation

$x \in \Omega: \quad c(T) \frac{\partial T(x, t)}{\partial t}=\nabla[\lambda(T) \nabla T(x, t)]+Q \frac{\partial f_{S}(x, t)}{\partial t}$

where $c(T)$ is a volumetric specific heat of casting material, $\lambda(T)$ is a thermal conductivity, $Q$ is a volumetric latent heat, $T=T(x, t), f_{S}=f_{S}(x, t)$ denote the temperature and the local volumetric fraction of solid state at the neighborhood of the point considered. The different forms of equation (1) appear at the stage of solidification rate $\partial f_{S} / \partial t$ computations (e.g. [7]).

Let us denote the temperatures corresponding to the beginning and the end of solidification process by $T_{L}$ and $T_{S}$ at the same time we assume the knowledge of temperaturedependent function $f_{S}$ for the interval $\left[T_{S}, T_{L}\right]$ and then

* THE SILESIAN UNIVERSITY OF TECHNOLOGY, FACULTY OF MECHANICAL ENGINEERING, KONARSKIEGO 18A, 44-100 GLIWICE, POLAND

** UNIVERSITY OF OCCUPATIONAL SAFETY MANAGEMENT IN KATOWICE, 8 BANKOWA STR., 40-007 KATOWICE, POLAND

\# Corresponding author: Ewa.Majchrzak@polsl.pl 


$$
\frac{\partial f_{S}(x, t)}{\partial t}=\frac{\mathrm{d} f_{S}}{\mathrm{~d} T} \frac{\partial T(x, t)}{\partial t}
$$

Introducing this formula to energy equation (1) one obtains

$$
x \in \Omega: \quad C(T) \frac{\partial T(x, t)}{\partial t}=\nabla[\lambda(T) \nabla T(x, t)]
$$

where $C(T)=c(T)-Q \mathrm{~d} f_{S} / \mathrm{d} T$ is called 'a substitute thermal capacity'. This parameter can be defined in the different ways, but one can see that for $T<T_{\mathrm{S}}: f_{S}=0$, while for $T>T_{L}: f_{S}=$ 0 and the derivative $\mathrm{d} f_{S} / \mathrm{d} T=0$. Summing up, the following definition of substitute thermal capacity can be accepted [18]

$$
C(T)=\left\{\begin{array}{lr}
c_{L} & T>T_{L} \\
c_{P}-Q \frac{\mathrm{d} f_{S}}{\mathrm{~d} T} & T_{S} \leq T \leq T_{L} \\
c_{S} & T<T_{S}
\end{array}\right.
$$

or, because $f_{L}=1-f_{S}$

$$
C(T)=\left\{\begin{array}{lr}
c_{L} & T>T_{L} \\
c_{P}+Q \frac{\mathrm{d} f_{L}}{\mathrm{~d} T} & T_{S} \leq T \leq T_{L} \\
c_{S} & T<T_{S}
\end{array}\right.
$$

where $c_{L}, c_{P}, c_{S}$ are the volumetric specific heats of molten metal, mushy zone and solid state sub-domains and one can use the equation (3) as the model of thermal processes proceeding in the whole, conventionally homogeneous, casting domain. It is the reason that the approach presented is called 'a one domain method'. Parameter $C(T)$ can be also defined directly without knowing the function $f_{S}(x, t)$ (e.g. [8]). In this case, the information concerning the change of alloy physical enthalpy between $T_{S}$ and $T_{L}$ should be used. Such an approach is presented in this paper.

The typical mathematical description of the real foundry technology requires the supplement of equation (3) by the equation determining the course of thermal processes in a mould sub-domain, this means

$$
x \in \Omega_{m}: \quad c_{m}(T) \frac{\partial T_{m}(x, t)}{\partial t}=\nabla\left[\lambda_{m}(T) \nabla T_{m}(x, t)\right]
$$

where the index $m$ identifies the mould sub-domain, the nonhomogeneous mould can be also considered.

On the external surface of mould the following boundary condition

$$
x \in \Gamma_{0}: \quad-\lambda_{m} \frac{\partial T_{m}(x, t)}{\partial n}=\alpha\left[T_{m}(x, t)-T_{a}\right]
$$

is, as a rule, accepted. Here $\alpha$ is a heat transfer coefficient, $T_{\alpha}$ is an ambient temperature, $\partial / \partial n$ denotes a normal derivative.
On the contact surface between casting and mould the continuity condition is given

$x \in \Gamma_{c}: \quad-\lambda \frac{\partial T(x, t)}{\partial n}=\frac{T(x, t)-T_{m}(x, t)}{R(x, t)}=-\lambda_{m} \frac{\partial T_{m}(x, t)}{\partial n}$

where $R$ is a thermal resistance. For $R=0$ (a such assumption can be done in the case of sand mix mould) the last equation takes a form

$$
\begin{aligned}
& x \in \Gamma_{c}: \quad\left\{\begin{array}{l}
-\lambda \frac{\partial T(x, t)}{\partial n}=-\lambda_{m} \frac{\partial T_{m}(x, t)}{\partial n} \\
T(x, t)=T_{m}(x, t)
\end{array}\right. \\
& t=0: \quad T(x, 0)=T_{0}(x), \quad T_{m}(x, 0)=T_{m 0}(x)
\end{aligned}
$$

The mathematical model presented above can be more complicated. For example, one can consider the convectional component of heat transfer which appear in the molten metal sub-domain, e.g. [19].

\section{Substitute thermal capacity of material considered}

In the case of typical binary alloys (e.g. Al-Si, Cu$\mathrm{Zn}$ ) the good results of solidification process modeling can be obtained using the approximation of STC in the form of bell-type function [20], however the process of cast iron solidification is more complex. During the process discussed the austenitic and eutectic phases are separated and both the austenite $Q_{a u s}$ and eutectic $Q_{e u}$ volumetric latent heats should be taken into account. The preliminary studies have shown that the favored is the division of $Q_{\text {aus }}$ into two components $Q_{\text {ausl }}, Q_{\text {aus } 2}$ and to define the STC in the following way

$C(T)= \begin{cases}c_{L}, & T \geq T_{L} \\ a_{1}+a_{2} T+a_{3} T^{2}+a_{4} T^{3}+a_{5} T^{4}, & T_{A} \leq T<T_{L} \\ c_{A E}, & T_{E} \leq T<T_{A}(11) \\ b_{1}+b_{2} T+b_{3} T^{2}+b_{4} T^{3}+b_{5} T^{4}, & T_{S} \leq T<T_{E} \\ c_{S}, & T<T_{S}\end{cases}$

where $T_{L}, T_{A}, T_{E}, T_{S}$ (Figure 1) are the border temperatures, $a_{k}, b_{k}, k=1,2, \ldots, 5$ are the unknown coefficients, while

$$
c_{A E}=\frac{c_{L}+c_{S}}{2}+\frac{Q_{a u s 2}}{T_{A}-T_{E}}
$$

The parameters of polynomials appearing in equation (11) should be determined in this way in order to assure the continuity and differentiability of $C(T)$, additionally the integration of polynomials in the selected temperature intervals must correspond to the thermal effects (changes of alloy physical enthalpy) connected with the solidification and cooling processes [20]. 
The very tedious mathematical manipulations finally give $a_{1}=\frac{c_{A E} T_{L}-c_{L} T_{A}}{T_{L}-T_{A}}+\frac{\left(c_{L}-c_{A E}\right) T_{L} T_{A}\left(T_{L}+T_{A}\right)}{\left(T_{L}-T_{A}\right)^{3}}+\frac{30 T_{L}^{2} T_{A}^{2} Q_{a u s 1}}{\left(T_{L}-T_{A}\right)^{5}}$ $a_{2}=-\frac{6\left(c_{L}-c_{A E}\right) T_{L} T_{A}}{\left(T_{L}-T_{A}\right)^{3}}-\frac{60 T_{L} T_{A}\left(T_{L}+T_{A}\right) Q_{a u s 1}}{\left(T_{L}-T_{A}\right)^{5}}$

$a_{3}=\frac{3\left(c_{L}-c_{A E}\right)\left(T_{L}+T_{A}\right)}{\left(T_{L}-T_{A}\right)^{3}}+\frac{30\left(T_{L}^{2}+4 T_{L} T_{A}+T_{A}^{2}\right) Q_{a u s 1}}{\left(T_{L}-T_{A}\right)^{5}}$

$a_{4}=-\frac{2\left(c_{L}-c_{A E}\right)}{\left(T_{L}-T_{A}\right)^{3}}-\frac{60\left(T_{L}+T_{A}\right) Q_{a u s 1}}{\left(T_{L}-T_{A}\right)^{5}}$

$a_{5}=\frac{30 Q_{a u s 1}}{\left(T_{L}-T_{A}\right)^{5}}$

and

$b_{1}=\frac{c_{S} T_{E}-c_{A E} T_{S}}{T_{E}-T_{S}}+\frac{\left(c_{A E}-c_{S}\right) T_{E} T_{S}\left(T_{E}+T_{S}\right)}{\left(T_{E}-T_{S}\right)^{3}}+\frac{30 T_{E}^{2} T_{S}^{2} Q_{e u}}{\left(T_{E}-T_{S}\right)^{5}}$

$b_{2}=-\frac{6\left(c_{A E}-c_{S}\right) T_{E} T_{S}}{\left(T_{E}-T_{S}\right)^{3}}-\frac{60 T_{E} T_{S}\left(T_{E}+T_{S}\right) Q_{e u}}{\left(T_{E}-T_{S}\right)^{5}}$

$b_{3}=\frac{3\left(c_{A E}-c_{S}\right)\left(T_{E}+T_{S}\right)}{\left(T_{E}-T_{S}\right)^{3}}+\frac{30\left(T_{E}^{2}+4 T_{E} T_{S}+T_{S}^{2}\right) Q_{e u}}{\left(T_{E}-T_{S}\right)^{5}}$

$b_{4}=-\frac{2\left(c_{A E}-c_{S}\right)}{\left(T_{E}-T_{S}\right)^{3}}-\frac{60\left(T_{E}+T_{S}\right) Q_{e u}}{\left(T_{E}-T_{S}\right)^{5}}$

$b_{5}=\frac{30 Q_{e u}}{\left(T_{E}-T_{S}\right)^{5}}$

The border temperatures and volumetric specific heats are assumed to be known and then the values of the polynomials parameters are dependent on the latent heat components $Q_{e u}$, $Q_{\text {aus } 1}$ and $Q_{\text {aus } 2}$.

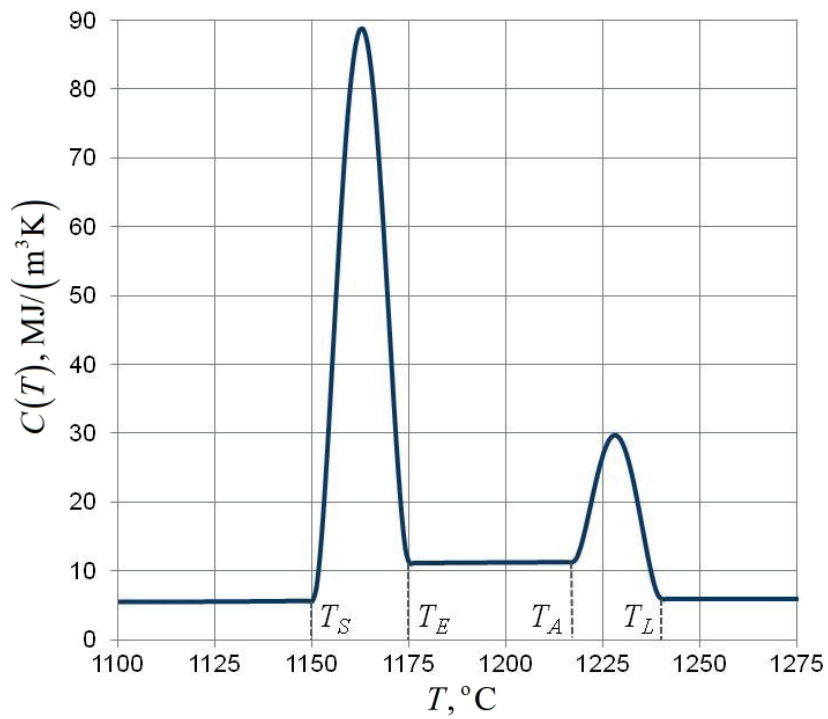

Fig. 1. Substitute thermal capacity

\section{Estimation of STC parameters using the gradient method}

The parameters determining the course of cast iron solidification will be denoted as $p_{e}, e=1,2, \ldots, E$. In the case considered $p_{1}=Q_{e u}, p_{2}=Q_{a u s 1}, p_{3}=Q_{a u s 2}$. The solution of the inverse problem requires an additional information concerning the course of the process and it is, as a rule, the knowledge of cooling/ heating curves at the set of points selected from the casting -mould domain. The least squares criterion is introduced $[10,11,21,22]$

$$
S=\frac{1}{M F} \sum_{i=1}^{M} \sum_{f=1}^{F}\left(T_{i}^{f}-T_{d i}^{f}\right)^{2} \rightarrow \mathrm{MIN}
$$

where $T_{d i}^{f}, T_{i}^{f}=T\left(x_{i}, t^{f}\right)$ are the measured values of temperature at the point $x_{i}$ and time $t^{f}$ and the temperatures at the same point and time found on the basis of numerical solution for a priori assumed values of the process parameters. The application of gradient method, e.g. [11, 23, 24] requires the differentiation of criterion (15) with respect to unknown parameters $p_{e}, e=1,2, \ldots, E$ and next the necessary condition of multivariable function extreme is taken into account

$$
\frac{\partial S}{\partial p_{e}}=\frac{2}{M F} \sum_{i=1}^{M} \sum_{f=1}^{F}\left(T_{i}^{f}-T_{d i}^{f}\right)\left(Z_{i e}^{f}\right)^{k}=0
$$

where

$$
\left(Z_{i e}^{f}\right)^{k}=\left.\frac{\partial T_{i}^{f}}{\partial p_{e}}\right|_{p_{e}=p_{e}^{k}}
$$

are the sensitivity coefficients, $k$ is the iteration number, at the same time the initial values $p_{e}{ }^{0}$ are the arbitrary assumed, while $p_{e}{ }^{k}$ for $k>0$ result from the previous iteration.

Function $T_{i}^{f}$ is expanded into the Taylor series taking into account the first derivative, this means

$$
T_{i}^{f}=\left(T_{i}^{f}\right)^{k}+\sum_{l=1}^{E}\left(Z_{i l}^{f}\right)^{k} \Delta p_{l}^{k}
$$

where

$$
\Delta p_{l}^{k}=p_{l}^{k+1}-p_{l}^{k}
$$

Introducing the formula (18) to the equation (16) for $e=1,2, \ldots, E$ one obtains

$$
\sum_{i=1}^{M} \sum_{f=1}^{F} \sum_{l=1}^{E}\left(Z_{i l}^{f}\right)^{k}\left(Z_{i e}^{f}\right)^{k} \Delta p_{l}^{k}=\sum_{i=1}^{M} \sum_{f=1}^{F}\left[T_{d i}^{f}-\left(T_{i}^{f}\right)^{k}\right]\left(Z_{i e}^{f}\right)^{k}
$$

System of equations (20) allows one to determine the values $\Delta p_{e}{ }^{k}$ and next to find the new values of parameters $p_{e}{ }^{k+1}$

$$
p_{e}^{k+1}=p_{e}^{k}+\Delta p_{e}^{k}, \quad e=1,2, \ldots, E
$$

The iteration process is completed when the assumed 'stop criterion' is satisfied. The quickness of getting to the 
solution depends on the position of start point $p_{e}{ }^{0}, e=1,2$, $\ldots, E$. Because the inverse problems belong to the group of ill posed ones, so the computations not always finish with success and sometimes it is also connected with the choice of start point.

To find the sensitivity coefficients (17) the sensitivity models with respect to parameters $p_{e}$ must be solved. The sensitivity models result from the differentiation of energy equations and boundary-initial conditions with respect to the parameter considered (direct approach [25-27]). In order to simplify the form of sensitivity equation we assume that the thermal conductivities of casting and mould material and also the mould volumetric specific heat can be treated as the constant values. So, one obtains the following additional boundary-initial problems

$x \in \Omega: C(T) \frac{\partial Z_{e}(x, t)}{\partial t}=\lambda \nabla^{2} Z_{e}(x, t)-\frac{\partial C(T)}{\partial p_{e}} \frac{\partial T(x, t)}{\partial t}$

$x \in \Omega_{m}: c_{m} \frac{\partial Z_{m e}(x, t)}{\partial t}=\lambda_{m} \nabla^{2} Z_{m e}(x, t)$

$x \in \Gamma_{c}:\left\{\begin{array}{c}-\lambda \frac{\partial Z_{e}(x, t)}{\partial n}=-\lambda_{m} \frac{\partial Z_{m e}(x, t)}{\partial n} \\ Z_{e}(x, t)=Z_{m e}(x, t)\end{array}\right.$

$x \in \Gamma_{0}:-\lambda_{m} \frac{\partial Z_{m e}(x, t)}{\partial n}=\alpha Z_{m e}(x, t)$

$t=0: \quad Z_{e}(x, 0)=0, Z_{m e}(x, 0)=0$

Differentiation of STC with respect to $p_{1}, p_{2}, p_{3}$ gives

$$
\frac{\partial C(T)}{\partial p_{1}}= \begin{cases}0, & T \geq T_{L} \\ \frac{\partial a_{1}}{\partial p_{1}}+\frac{\partial a_{2}}{\partial p_{1}} T+\frac{\partial a_{3}}{\partial p_{1}} T^{2}+\frac{\partial a_{4}}{\partial p_{1}} T^{3}+\frac{\partial a_{5}}{\partial p_{1}} T^{4}+ & T_{A} \leq T<T_{L} \\ \left(a_{2}+2 a_{3} T+3 a_{4} T^{2}+4 a_{5} T^{3}\right) Z_{1}, & T_{E} \leq T<T_{A} \\ 0, & T_{S} \leq T<T_{E} \\ 0, & T<T_{S}\end{cases}
$$

and

$$
\frac{\partial C(T)}{\partial p_{2}}=\left\{\begin{array}{lc}
0, & T \geq T_{L} \\
0, & T_{A} \leq T<T_{L} \\
\frac{1}{T_{A}-T_{E}}, & T_{E} \leq T<T_{A} \\
0, & T_{S} \leq T<T_{E} \\
0, & T<T_{S}
\end{array}\right.
$$

while

$$
\frac{\partial C(T)}{\partial p_{3}}=\left\{\begin{array}{lc}
0, & T \geq T_{L} \\
0, & T_{A} \leq T<T_{L} \\
0, & T_{E} \leq T<T_{A} \\
\frac{\partial b_{1}}{\partial p_{3}}+\frac{\partial b_{2}}{\partial p_{3}} T+\frac{\partial b_{3}}{\partial p_{3}} T^{2}+\frac{\partial b_{4}}{\partial p_{3}} T^{3}+\frac{\partial b_{5}}{\partial p_{3}} T^{4}+ & T_{S} \leq T<T_{E} \\
\left(b_{2}+2 b_{3} T+3 b_{4} T^{2}+4 b_{5} T^{3}\right) Z_{3}, & \\
0, & T<T_{S}
\end{array}\right.
$$

Using the formulas (13) and (14) one can find the derivatives $\partial a_{k} / \partial p_{1}$ and $\partial b_{k} / \partial p_{3}$, in particular

$$
\begin{aligned}
& \frac{\partial a_{1}}{\partial p_{e}}=\frac{30 T_{L}^{2} T_{A}^{2}}{\left(T_{L}-T_{A}\right)^{5}}, \frac{\partial a_{2}}{\partial p_{e}}=-\frac{60 T_{L} T_{A}\left(T_{L}+T_{A}\right)}{\left(T_{L}-T_{A}\right)^{5}} \\
& \frac{\partial a_{3}}{\partial p_{e}}=-\frac{30\left(T_{L}^{2}+4 T_{L} T_{A}+T_{A}^{2}\right)}{\left(T_{L}-T_{A}\right)^{5}} \\
& \frac{\partial a_{4}}{\partial p_{e}}=-\frac{60\left(T_{L}+T_{A}\right)}{\left(T_{L}-T_{A}\right)^{5}}, \frac{\partial a_{5}}{\partial p_{e}}=\frac{30}{\left(T_{L}-T_{A}\right)^{5}}
\end{aligned}
$$

and

$$
\begin{aligned}
& \frac{\partial b_{1}}{\partial p_{e}}=\frac{30 T_{E}^{2} T_{S}^{2}}{\left(T_{E}-T_{S}\right)^{5}}, \frac{\partial b_{2}}{\partial p_{e}}=-\frac{60 T_{E} T_{S}\left(T_{E}+T_{S}\right)}{\left(T_{E}-T_{S}\right)^{5}} \\
& \frac{\partial b_{3}}{\partial p_{e}}=-\frac{30\left(T_{E}^{2}+4 T_{E} T_{S}+T_{S}^{2}\right)}{\left(T_{E}-T_{S}\right)^{5}} \\
& \frac{\partial b_{4}}{\partial p_{e}}=-\frac{60\left(T_{E}+T_{S}\right)}{\left(T_{E}-T_{S}\right)^{5}}, \frac{\partial b_{5}}{\partial p_{e}}=\frac{30}{\left(T_{E}-T_{S}\right)^{5}}
\end{aligned}
$$

From the practical point of view both the sensitivity models and the basic one are similar and at the stage of numerical computations almost the same computer programs can be used.

To verify the correctness and effectiveness of numerical algorithm the following task has been solved. The cast iron plate shown in Figure 2 is produced in the typical sand mould (a problem is treated as $2 \mathrm{D}$ one).
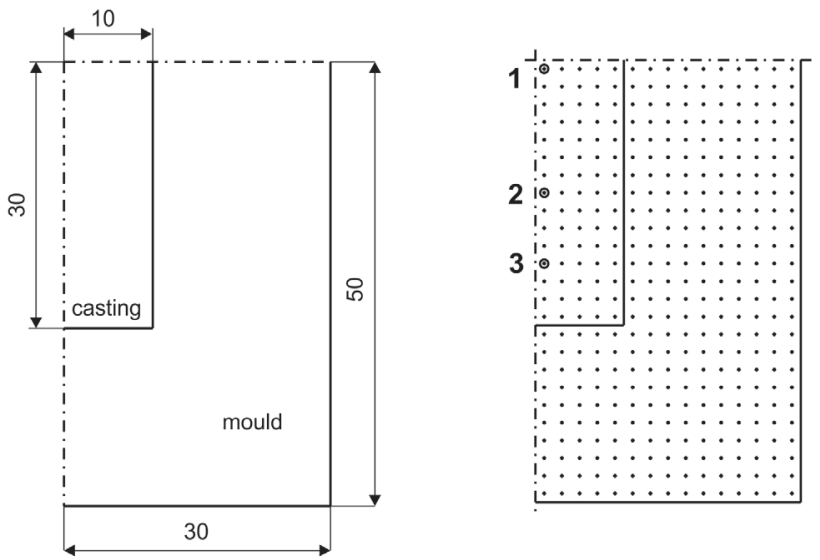

Fig. 2. Casting - mould system and the differential mesh 
Thermophysical parameters of subdomains are as follows: $\lambda=30 \mathrm{~W} /(\mathrm{m} \mathrm{K}), \lambda_{m}=1 \mathrm{~W} /(\mathrm{m} \mathrm{K}), c_{L}=5.88 \mathrm{MJ} /\left(\mathrm{m}^{3} \mathrm{~K}\right)$, $c_{S}=5.4 \mathrm{MJ} /\left(\mathrm{m}^{3} \mathrm{~K}\right), c_{m}=1.75 \mathrm{MJ} /\left(\mathrm{m}^{3} \mathrm{~K}\right)$, while $Q_{\text {aus } 1}=937.2$ $\mathrm{MJ} / \mathrm{m}^{3}, Q_{\text {aus } 2}=397.6 \mathrm{MJ} / \mathrm{m}^{3}, Q_{e u}=582.2 \mathrm{MJ} / \mathrm{m}^{3}$ (estimated parameters). Border temperatures are equal to $T_{L}=1250^{\circ} \mathrm{C}$, $T_{S}=1110^{\circ} \mathrm{C}, T_{A}=1200^{\circ} \mathrm{C}, T_{E}=1130^{\circ} \mathrm{C}, T_{0}=1300^{\circ} \mathrm{C}$ and $\mathrm{T}_{m} 0$ $=20^{\circ} \mathrm{C}$. The solution of the basic problem played the role of 'measured temperatures' at the points 1,2, 3 corresponding to the sensors positions. The obtained cooling curves at the points 1, 2, 3 are shown in Figure 3. The computations, as previously mentioned, have been performed using the explicit variant of the FDM.

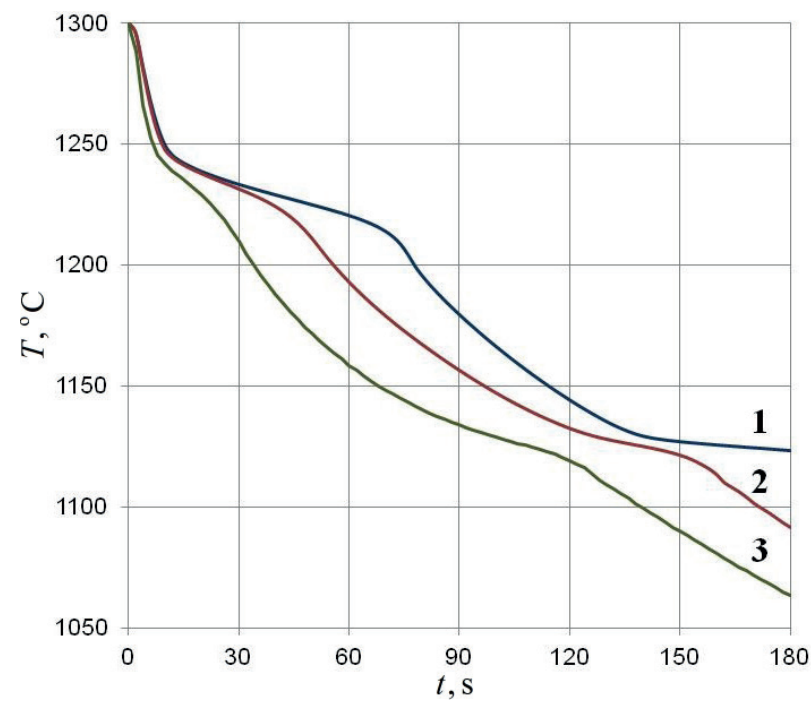

Fig. 3. Cooling curves

The identification problem has been solved using the gradient method. The start point corresponded to the values $Q_{a u s 1}, Q_{a u s 2}, Q_{e u}=0 \mathrm{MJ} / \mathrm{m}^{3}$, the course of iteration process is shown in Figure 4.

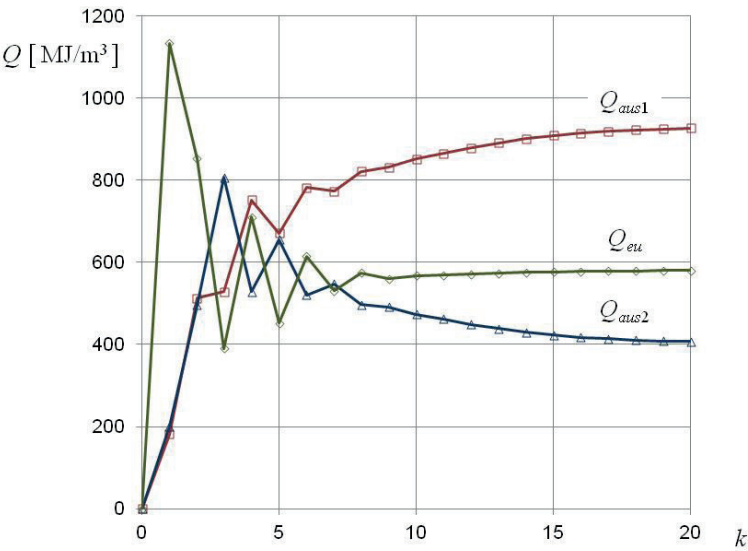

Fig. 4. Course of iteration process
One can see, that the computational process is convergent although the start point is far from the final one. The results after 20 loops, this means $Q_{\text {aus } 1}{ }^{20}=931.41 \mathrm{MJ} / \mathrm{m} 3, Q_{\text {aus } 2}{ }^{20}=$ $401.52 \mathrm{MJ} / \mathrm{m}^{3}, Q_{e u}{ }^{20}=584.63 \mathrm{MJ} / \mathrm{m}^{3}$ are close to the values assumed at the stage of the basic problem solution. The same, as previously, problem has been also solved using the disturbed input data (cooling curves) (e.g. [28]) and the results obtained have been practically the same.

\section{Application of measurements}

Good results of STC identification on the basis of calculated cooling curves confirmed the correctness of the algorithm prepared and used numerical procedures. The next stage of research was connected with the application of the real measurements. So, the castings in the shape of $\mathrm{X}$ and $\mathrm{L}$ hot spots have been made (gray iron $3.21 \% \mathrm{C}, 1.9 \%$ $\mathrm{Si}$ ). The test stand for temperature measurements is shown in Figure 5.

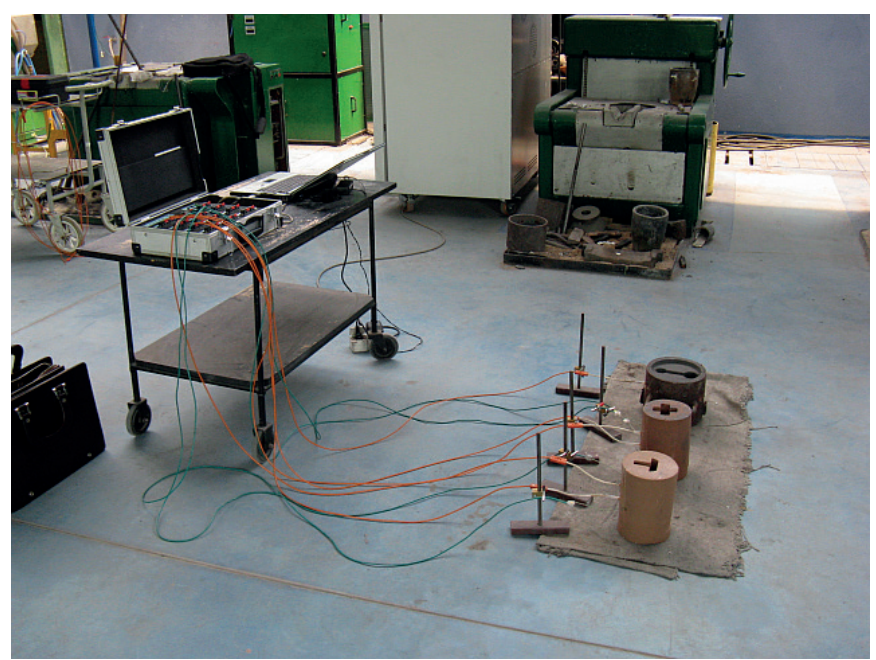

Fig. 5. Measuring stand

As an example, the results concerning the system shown in Figure 6 will be below discussed. In particular, the identification of unknown parameters on the basis of cooling curve measured at the point 3 (Fig. 6) will be presented. In Figure 7 the measured temperature history and the numerical solution obtained for the optimal values of $Q_{a u s}, Q_{a u s 2}$ and $Q_{e u}$ are shown. They are equal to $Q_{\text {aus } 1}=469.6 \mathrm{MJ} / \mathrm{m} 3, Q_{\text {aus } 2}=$ $413.6 \mathrm{MJ} / \mathrm{m}^{3}, Q_{e u}=1232 \mathrm{MJ} / \mathrm{m}^{3}$, while the value of functional (15) is equal to $S=25.2$. 


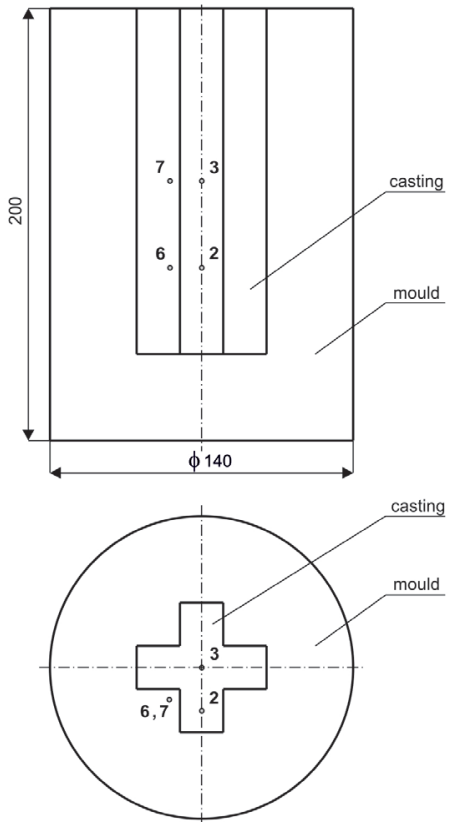

Fig. 6. X type hot spot

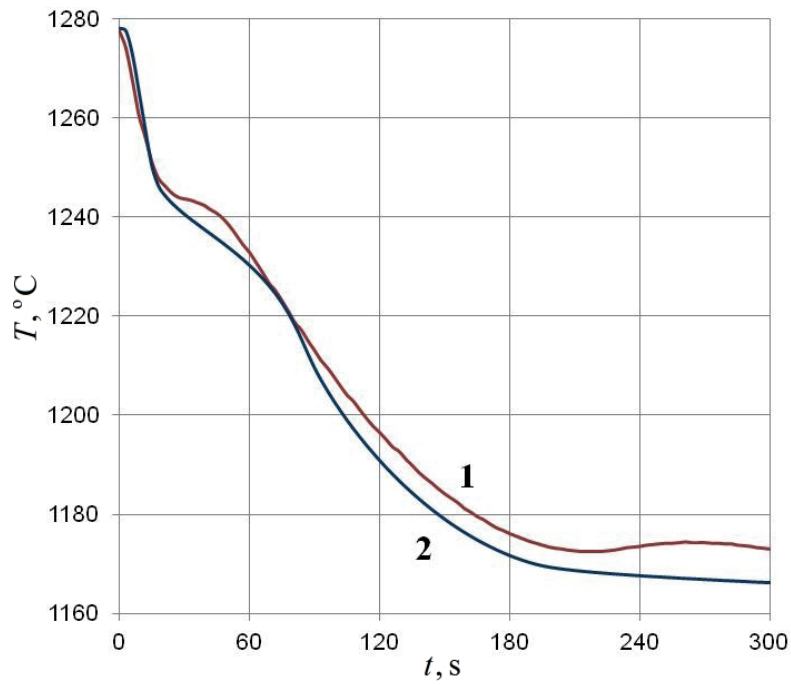

Fig. 7. Measured (1) and calculated (2) cooling curves at the point 3

In this paper only the very small part of the results obtained is presented. Among others, the different shapes of castings have been considered and the different numbers of sensors located in the domains of casting and mould have been taken into account. The results of parameters estimation are not the same, but the deviations are not big and quite acceptable. In the cases considered the iteration process is quickly convergent.

\section{Conclusions}

At the stage of alloys solidification modeling using the macroscale approach the application of the one domain method seems to be very effective way of process description both from the mathematical and numerical points of view. For a large number of alloys the function determining the course of parameter discussed can be constructed in a simple way e.g. in the form of bell-type function 'stretched' between border temperatures $T_{L}$ and $T_{S}$. In the case of cast iron the situation is more complicated - it results from the complexity of solidification process. Preliminary studies led to the conclusion that the STC can be described by the composition of two bell-type functions and the constant value. The successive components (assuming the knowledge of the border temperatures) are dependent on the quantities $Q_{a u s 1}, Q_{a u s 2}$ and $Q_{e u}$. They are determined using the methods of inverse problems solutions, in particular the gradient method has been applied. To check the correctness and effectiveness of numerical procedures developed, the examples for which the solutions of the direct problems play a role of measurements have been done. Next, the series of castings has been made and the additional information necessary to solve the identification problem resulted from the measured cooling/heating curves at the set of points selected from the casting-mould system. The results of parameters estimation are fully satisfactory.

\section{REFERENCES}

[1] M. Muhieddine, E. Canot, R. March, Various approaches for solving problems in heat conduction with phase change, International Journal on Finite 6 (1) 1-20 (2009).

[2] B. Mochnacki, E. Majchrzak, Numerical modeling of casting solidification using generalized finite difference method, Materials Science Forum 638-642, 2676-2681 (2010).

[3] W. Piekarska, M. Kubiak, Three-dimensional model for numerical analysis of thermal phenomena in laser-arc hybrid welding process, International Journal of Heat and Mass Transfer 54, 23-24, 4966-4974 (2011).

[4] G. Comini, L. Del Giudice, Thermal aspects of cryosurgery, Journao of Heat Transfer 98, 543-549 (1976).

[5] M. Muhieddine, E. Canot, R. March, Heat transfer modeling in saturated porous media and identification of the thermophysical properties of the soil by inverse problem, Applied Numerical Mathematics 62, 1026-1040 (2012).

[6] B. Mochnacki, R. Szopa, Identification of alloy latent heat using the data of thermal and differential analysis, Journal of Theoretical and Applied Mechanics 49, 4, 1019-1028 (2011).

[7] B. Mochnacki, E. Majchrzak, Identification of macro and micro parameters in solidification model, Bulletin of the Polish Academy of Sciences, Technical Sciences 55, 1, 107113 (2007).

[8] E. Majchrzak, B. Mochnacki, J.S. Suchy, Identification of substitute thermal capacity of solidifying alloy, Journal of Theoretical and Applied Mechanics 46, 2, 257-268 (2008).

[9] E. Hetmaniok, I. Nowak, D. Słota, A. Zielonka, Determination of optimal parameters for the immune algorithm used for solving inverse heat conduction problems with and without a phase change, Numerical Heat Transfer Part B Fundamentals 62, 6, 462-478 (2012).

[10] O.M. Alifanov, Inverse heat transfer problems, SpringerVerlag (1994).

[11] K. Kurpisz, A. Nowak, Inverse Thermal Problems, Southampton, Boston: Computational Mechanics Publications (1995).

[12] Ch.H. Huang, J.Y. Yan, An inverse problem in simultaneously measuring temperature-dependent thermal conductivity and 
heat capacity, International Journal, of Heat and Mass Transfer 38, 18, 3433-3441 (1995)

[13] R. Grzymkowski, D. Słota, One-phase inverse Stefan problem solved by Adomian decomposition method, Computers \& Mathematics with Applications 51, 1, 33-40 (2006).

[14] V. Grozdani, Finite-difference methods for simulating the solidification of castings, Materials and Technology 43, 5, 233-237 (2009).

[15] A. Rouboa, E. Monteiro, Heat transfer in multi-block grid during solidification: performance of finite differences and finite volume method, Journal of Materials Processing Technology 204, 451-458 (2008).

[16] A.A. Samarski, The theory of difference schemes, Marcel Dekker, Inc., New York, Basel (2001).

[17] M. Ciesielski, A multiscale approach to numerical modeling of solidification, International Journal for Multiscale Computational Engineering 8, 3, 251-257 (2010).

[18] E. Majchrzak, J. Mendakiewicz, Numerical analysis of cast iron solidification process, Journal of Materials Processing Technology 53, 1-2, 285-292 (1995).

[19] A. Reikher, K.M. Pillai, A fast simulation of transient metal flow and solidification in a narrow channel. Part I: Model development using lubrication approximation, International Journal of Heat and Mass Transfer 60, 797-805 (2013).

[20] E. Majchrzak, M. Dziewoński, G. Kałuża, Numerical algorithm of cast steel latent heat identification, Journal of
Achievements of Materials and Manufacturing Engineering 22, 1, 61-64 (2007).

[21] E. Hensel, Inverse theory and applications for engineers, Prentice Hall, New Yersey (1991).

[22] M.N. Ozisik, H.R.B. Orlande, Inverse heat transfer: Fundamentals and applications, Taylor and Francis, Pennsylvania, (1999).

[23] I. Daubechies, M. Fornasier, I. Loris, Accelerated projected gradient method for linear inverse problems with sparsity constraints, Journal of Fourier Analysis and Applications 14, 764-792 (2008)

[24] A. Beck, M. Teboulle, A fast iterative shrinkage-thresholding algorithm for linear inverse problems, SIAM Journal on Imaging Sciences 2, 1, 183-202 (2009).

[25] R. Szopa, Sensitivity analysis and inverse problems in the thermal theory of foundry processes, Publications of the Częstochowa University of Technology, Częstochowa (2006) - in Polish.

[26] M. Kleiber, Parameter sensitivity, J.Wiley \& Sons Ltd., Chichester (1997).

[27] K. Dems, B. Rousselet, Sensitivity analysis for transient heat conduction in a solid body, Structural Optimization 17, 36-45 (1999).

[28] R. Grzymkowski, Inverse problems in the thermal theory of foundry processes. Publ. of the Silesian Univ. of Techn., Mechanics 105, Gliwice, 1991 (in Polish). 
